\title{
$N$-Bright-Dark Soliton Solution to a Semi-Discrete Vector Nonlinear Schrödinger Equation
}

\author{
Bao-Feng FENG ${ }^{\dagger}$ and Yasuhiro OHTA $\ddagger$ \\ † School of Mathematical and Statistical Sciences, The University of Texas Rio Grande Valley, \\ Edinburg, TX 78539, USA \\ E-mail: baofeng.feng@utrgv.edu \\ $\ddagger$ Department of Mathematics, Kobe University, Rokko, Kobe 657-8501, Japan \\ E-mail: ohta@math.kobe-u.ac.jp
}

Received April 25, 2017, in final form September 03, 2017; Published online September 06, 2017

https://doi.org/10.3842/SIGMA.2017.071

\begin{abstract}
In this paper, a general bright-dark soliton solution in the form of Pfaffian is constructed for an integrable semi-discrete vector NLS equation via Hirota's bilinear method. One- and two-bright-dark soliton solutions are explicitly presented for two-component semidiscrete NLS equation; two-bright-one-dark, and one-bright-two-dark soliton solutions are also given explicitly for three-component semi-discrete NLS equation. The asymptotic behavior is analysed for two-soliton solutions.
\end{abstract}

Key words: bright-dark soliton; semi-discrete vector NLS equation; Hirota's bilinear method; Pfaffian

2010 Mathematics Subject Classification: 39A10; 35Q55

\section{Introduction}

The nonlinear Schrödinger (NLS) equation

$$
\mathrm{i} u_{t}=u_{x x}+2 \sigma|u|^{2} u
$$

is a generic model equation describing the evolution of small amplitude and slowly varying wave packets in weakly nonlinear media $[2,6,8,18,23]$. It arises in a variety of physical contexts such as nonlinear optics [19, 20], Bose-Einstein condensates [14], water waves [13] and plasma physics [48]. The integrability, as well as the bright-soliton solution in the focusing case $(\sigma=1)$, was found by Zakharov and Shabat $[49,50]$. The dark soliton was found in the defocusing NLS equation $(\sigma=-1)[20,25,42]$, and was observed experimentally in [24, 46].

The integrable discretization of nonlinear Schrödinger equation

$$
\mathrm{i} q_{n, t}=\left(1+\sigma\left|q_{n}\right|^{2}\right)\left(q_{n+1}+q_{n-1}\right)
$$

was originally derived by Ablowitz and Ladik [3, 4], so it is also called the Ablowitz-Ladik (AL) lattice equation. Similar to the continuous case, it is known that the AL lattice equation, by Hirota's bilinear method, admits the bright soliton solution for the focusing case $(\sigma=1)$ [28, 41], also the dark soliton solution for the defocusing case $(\sigma=-1)$ [27]. The inverse scattering transform (IST) has been developed by several authors in the literature [1, 36, 43, 44]. The geometric construction of the AL lattice equation was given by Doliwa and Santini [15].

This paper is a contribution to the Special Issue on Symmetries and Integrability of Difference Equations. The full collection is available at http://www.emis.de/journals/SIGMA/SIDE12.html 
The coupled nonlinear Schrödinger equation

$$
\begin{aligned}
& \mathrm{i} u_{t}=u_{x x}+2\left(\sigma_{1}|u|^{2}+\sigma_{2}|v|^{2}\right) u, \\
& \mathrm{i} v_{t}=v_{x x}+2\left(\sigma_{1}|u|^{2}+\sigma_{2}|v|^{2}\right) v,
\end{aligned}
$$

where $\sigma_{i}= \pm 1, i=1,2$, was firstly recognized being integrable by Yajima and Oikawa [47]. For the focusing-focusing case $\left(\sigma_{1}=\sigma_{2}=1\right)$, the system (1.1) solved by Manakov via inverse scattering transform (IST), admits the bright-bright soliton solution [26], so it is also called the Manakov system in the literature. For the defocusing-defocusing case, the Manakov system admits bright-dark and dark-dark soliton solution [35, 37, 38]. However, the focusing-defocusing Manakov system admits all types of soliton solutions such as bright-bright solitons, dark-dark soliton, and bright-dark solitons [22, 33, 45]. The Manakov system can be easily extended to a multi-component case, the so-called vector NLS equation. For the continuous vector NLS equation, the $N$-bright soliton solution was obtained in [16, 22, 51]; the general bright-dark and darkdark soliton solutions were obtained in $[16,17,25,34,45]$. The inverse scattering transform with nonvanishing boundary condition was solved by Prinari, Ablowitz and Biondini [35]. We should remark here that the problem of constructing exact soliton solutions to the vector NLS equation and proving their nonsingularity was settled by Dubrovin et al. in their landmark paper [16].

The semi-discrete coupled nonlinear Schrödinger equation

$$
\begin{aligned}
\mathrm{i} q_{n, t}^{(1)} & =\left(1+\sigma_{1}\left|q_{n}^{(1)}\right|^{2}+\sigma_{2}\left|q_{n}^{(2)}\right|^{2}\right)\left(q_{n+1}^{(1)}+q_{n-1}^{(1)}\right), \\
\mathrm{i} q_{n, t}^{(2)} & =\left(1+\sigma_{1}\left|q_{n}^{(1)}\right|^{2}+\sigma_{2}\left|q_{n}^{(2)}\right|^{2}\right)\left(q_{n+1}^{(2)}+q_{n-1}^{(2)}\right),
\end{aligned}
$$

where $\sigma_{i}= \pm 1, i=1,2$, is of importance both mathematically and physically. It was solved by the inverse scattering transform (IST) in [39, 40]. The general multi-soliton solution in terms of Pfaffians was found by one of the authors recently [31], which is of bright type for the focusing-focusing case $\left(\sigma_{1}=\sigma_{2}=1\right)$, and is of dark type for the defocusing-defocusing case $\left(\sigma_{1}=\sigma_{2}=-1\right)$. However, as far as we know, no general mixed-type (bright-dark) soliton solution is reported in the literature, which motivated the present study.

In the present paper, we consider a $M$-coupled semi-discrete NLS equation of all types

$$
\mathrm{i} q_{n, t}^{(j)}=\left(1+\sum_{\mu=1}^{M} \sigma_{\mu}\left|q_{n}^{(\mu)}\right|^{2}\right)\left(q_{n+1}^{(j)}+q_{n-1}^{(j)}\right), \quad j=1,2, \ldots, M,
$$

where $\sigma_{\mu}= \pm 1$ for $\mu=1, \ldots M$. For all-focusing case $\left(\sigma_{\mu}=1, \mu=1, \ldots, M\right)$, its general $N$-bright soliton solution and the interactions among solitons were studied in [5, 29]. However, in contrast with a complete list of the general $N$-soliton solution to the vector NLS equation [17], the mixed-type soliton solution of all possible nonlinearities (all possible values of $\sigma_{\mu}$ ) is missing. The aim of the present paper is to construct a general $N$-bright-dark soliton solution to the semidiscrete vector NLS equation. The rest of the paper is organized as follows. In Section 2, we provide a general bright-dark soliton solution in terms of Pfaffians to the semi-discrete vector NLS equation (1.3) and give a rigorous proof by the Pfaffian technique [21, 30, 32]. The one- and two-soliton solutions to two-coupled and three-coupled semi-discrete NLS equation are provided explicitly, respectively, in Section 3. We summarize the paper in Section 4 and present asymptotic analysis for two-soliton solution in Appendix A.

\section{General bright-dark soliton solution to semi-discrete vector NLS equation}

Let us consider a general soliton solution consisting of $m$-bright solitons and $(M-m)$-dark solitons to the semi-discrete vector NLS equation (1.3). To this end, we introduce the following 
dependent variable transformations

$$
q_{n}^{(j)}=\mathrm{i}^{n} \frac{g_{n}^{(j)}}{f_{n}}, \quad q_{n}^{(m+l)}=\rho_{l}\left(\mathrm{i} a_{l}\right)^{n} \frac{h_{n}^{(l)}}{f_{n}} e^{\omega_{l} t}
$$

where $j=1, \ldots, m, \omega_{l}=s\left(a_{l}-\bar{a}_{l}\right),\left|a_{l}\right|=1$ with $\bar{a}_{l}$ representing the complex conjugate of $a_{l}$, $l=1, \ldots, M-m$. Here, $f_{n}$ is a real-valued function, whereas, $g_{n}$ and $h_{n}$ are complex-valued functions. The transformations convert equation (1.3) into a set of bilinear equations as follows

$$
\begin{aligned}
& D_{t} g_{n}^{(j)} \cdot f_{n}=s\left(g_{n+1}^{(j)} f_{n-1}-g_{n-1}^{(j)} f_{n+1}\right), \quad j=1, \ldots, m, \\
& \left(D_{t}+\omega_{l}\right) h_{n}^{(l)} \cdot f_{n}=s\left(a_{l} h_{n+1}^{(l)} f_{n-1}-\bar{a}_{l} h_{n-1}^{(l)} f_{n+1}\right), \quad l=1, \ldots, M-m, \\
& s f_{n+1} f_{n-1}-f_{n}^{2}=\sum_{j=1}^{m} \sigma_{j}\left|g_{n}^{(j)}\right|^{2}+\sum_{l=1}^{M-m} \sigma_{l+m}\left|\rho_{l}\right|^{2}\left|h_{n}^{(l)}\right|^{2} .
\end{aligned}
$$

Here $s=1+\sum_{l=1}^{M-m} \sigma_{l+m}\left|\rho_{l}\right|^{2}$.

In what follows, we give a Pfaffian-type solution to the above bilinear equations.

Theorem 2.1. A set of bilinear equations (2.2) admit the following solutions in the form of Pfaffians

$$
\begin{aligned}
& f_{n}=\operatorname{Pf}\left(a_{1}, \ldots, a_{2 N}, b_{1}, \ldots, b_{2 N}\right), \\
& g_{n}^{(j)}=\operatorname{Pf}\left(d_{0}, \beta_{j}, a_{1}, \ldots, a_{2 N}, b_{1}, \ldots, b_{2 N}\right), \quad h_{n}^{(l)}=\operatorname{Pf}\left(c_{1}^{(l)}, \ldots, c_{2 N}^{(l)}, b_{1}, \ldots, b_{2 N}\right),
\end{aligned}
$$

with the elements of the Pfaffians defined as follows

$$
\begin{aligned}
& \operatorname{Pf}\left(a_{j}, a_{k}\right)_{n}=\frac{p_{j}-p_{k}}{p_{j} p_{k}-1} \varphi_{j}(n) \varphi_{k}(n), \quad \operatorname{Pf}\left(d_{0}, b_{j}\right)=\operatorname{Pf}\left(d_{0}, \beta_{l}\right)=0, \\
& \operatorname{Pf}\left(c_{j}^{(l)}, c_{k}^{(l)}\right)_{n}=\frac{p_{j}-p_{k}}{p_{j} p_{k}-1} \frac{p_{j}-a_{l}}{1-a_{l} p_{j}} \frac{p_{k}-a_{l}}{1-a_{l} p_{k}} \varphi_{j}(n) \varphi_{k}(n), \\
& \operatorname{Pf}\left(a_{j}, b_{k}\right)=\delta_{j k}, \quad \operatorname{Pf}\left(c_{j}^{(l)}, b_{k}\right)=\delta_{j k}, \quad \operatorname{Pf}\left(d_{l}, a_{k}\right)_{n}=p_{k}^{l} \varphi_{k}(n), \\
& \operatorname{Pf}\left(b_{j}, \beta_{l}\right)=\left\{\begin{array}{ll}
0, & 1 \leq j \leq N, \\
\alpha_{j-N}^{(l)}, & N+1 \leq j \leq 2 N,
\end{array} \quad \operatorname{Pf}\left(a_{j}, \beta_{l}\right)=0,\right. \\
& \operatorname{Pf}\left(b_{j}, b_{k}\right)= \begin{cases}b_{j k}, & 1 \leq j \leq N, N+1 \leq k \leq 2 N, \\
0, & \text { otherwise, }\end{cases}
\end{aligned}
$$

with

$$
b_{j k}=\frac{\sum_{l=1}^{m} \alpha_{j}^{(l)} \sigma_{l} \overline{\alpha_{k-N}^{(l)}}}{\left(p_{j}-p_{k}\right)\left(p_{j} p_{k}-1\right)\left(\frac{s}{p_{j} p_{k}}-\sum_{l=1}^{M-m} \frac{\sigma_{l+m}\left|\rho_{l}\right|^{2}\left(a_{l}-\bar{a}_{l}\right)^{2}}{\left(1-a_{l} p_{j}\right)\left(1-a_{l} p_{k}\right)\left(1-\bar{a}_{l} p_{j}\right)\left(1-\bar{a}_{l} p_{k}\right)}\right)},
$$

and $\varphi_{j}(n)=p_{j}^{n} e^{\eta_{j}}, \eta_{j}=s\left(p_{j}-p_{j}^{-1}\right) t+\eta_{j, 0}$ which satisfying $p_{j+N}=\bar{p}_{j}, \eta_{j+N, 0}=\bar{\eta}_{j, 0}$.

Proof. It can be shown that

$$
\begin{aligned}
& \frac{\mathrm{d}}{\mathrm{d} t} f_{n}=s \operatorname{Pf}\left(d_{-1}, d_{1}, \ldots\right)_{n}, \quad \text { where } \quad \operatorname{Pf}\left(d_{-1}, d_{1}\right) \equiv 0, \quad \operatorname{Pf}\left(d_{ \pm 1}, b_{j}\right) \equiv 0, \\
& f_{n+1}=\operatorname{Pf}\left(d_{0}, d_{1}, \ldots\right)_{n}, \quad \text { where } \quad \operatorname{Pf}\left(d_{0}, d_{1}\right) \equiv 1,
\end{aligned}
$$




$$
f_{n-1}=\operatorname{Pf}\left(d_{0}, d_{-1}, \ldots\right)_{n}, \quad \text { where } \quad \operatorname{Pf}\left(d_{0}, d_{-1}\right) \equiv 1,
$$

and

$$
\begin{aligned}
& \frac{\mathrm{d}}{\mathrm{d} t} g_{n}^{(j)}=s \operatorname{Pf}\left(d_{0}, d_{-1}, d_{1}, \beta_{j}, \ldots\right)_{n}, \\
& g_{n+1}^{(j)}=\operatorname{Pf}\left(d_{1}, \beta_{j}, \ldots\right)_{n}, \quad g_{n-1}^{(j)}=\operatorname{Pf}\left(d_{-1}, \beta_{j}, \ldots\right)_{n},
\end{aligned}
$$

where $\operatorname{Pf}\left(d_{0}, d_{1}, a_{1}, \ldots, a_{2 N}, b_{1}, \ldots, b_{2 N}\right)$ is abbreviated by $\operatorname{Pf}\left(d_{0}, d_{1}, \ldots\right)$, so as other similar Pfaffians. Thus, an algebraic identity of Pfaffian

$$
\begin{aligned}
& \operatorname{Pf}\left(d_{0}, d_{-1}, d_{1}, \beta_{j}, \ldots\right)_{n} \operatorname{Pf}(\ldots)_{n}=\operatorname{Pf}\left(d_{0}, d_{-1}, \ldots\right)_{n} \operatorname{Pf}\left(d_{1}, \beta_{j}, \ldots\right)_{n} \\
& \quad-\operatorname{Pf}\left(d_{0}, d_{1}, \ldots\right)_{n} \operatorname{Pf}\left(d_{-1}, \beta_{j}, \ldots\right)_{n}+\operatorname{Pf}\left(d_{0}, \beta_{j}, \ldots\right)_{n} \operatorname{Pf}\left(d_{-1}, d_{1}, \ldots\right)_{n}
\end{aligned}
$$

together with above Pfaffian relations gives

$$
\left(\frac{\mathrm{d}}{\mathrm{d} t} g_{n}^{(j)}\right) \times f_{n}=s g_{n+1}^{(j)} \times f_{n-1}-s g_{n-1}^{(j)} \times f_{n+1}+g_{n}^{(j)} \times\left(\frac{\mathrm{d}}{\mathrm{d} t} f_{n}\right),
$$

which is exactly the first bilinear equation. Next we prove the second bilinear equation. It can also be shown that

$$
\begin{aligned}
& h_{n}^{(l)}=\operatorname{Pf}\left(d_{0}, \bar{d}_{0}^{(l)}, \ldots\right)_{n}, \quad h_{n+1}^{(l)}=\bar{a}_{l} \operatorname{Pf}\left(d_{1}, \bar{d}_{0}^{(l)}, \ldots\right)_{n}, \quad h_{n-1}^{(l)}=a_{l} \operatorname{Pf}\left(d_{-1}, \bar{d}_{0}^{(l)}, \ldots\right)_{n}, \\
& \left(\frac{\mathrm{d}}{\mathrm{d} t}+s\left(a_{l}-\bar{a}_{l}\right)\right) h_{n}^{(l)}=s \operatorname{Pf}\left(d_{0}, d_{-1}, d_{1}, \bar{d}_{0}^{(l)}, \ldots\right)_{n},
\end{aligned}
$$

where

$$
\begin{aligned}
& \operatorname{Pf}\left(\bar{d}_{0}^{(l)}, a_{j}\right)=p_{j}^{n}\left(\frac{p_{j}-a_{l}}{1-a_{l} p_{j}}\right) e^{\eta_{j}}, \quad \operatorname{Pf}\left(\bar{d}_{0}^{(l)}, b_{j}\right)=0, \quad \operatorname{Pf}\left(d_{0}, \bar{d}_{0}^{(l)}\right)=1, \\
& \operatorname{Pf}\left(d_{-1}, \bar{d}_{0}^{(l)}\right)=\bar{a}_{l}, \quad \operatorname{Pf}\left(d_{1}, \bar{d}_{0}^{(l)}\right)=a_{l} .
\end{aligned}
$$

Therefore, an algebraic identity of Pfaffian

$$
\begin{aligned}
& \operatorname{Pf}\left(d_{0}, d_{-1}, d_{1}, \bar{d}_{0}^{(l)}, \ldots\right)_{n} \operatorname{Pf}(\ldots)_{n}=\operatorname{Pf}\left(d_{0}, d_{-1}, \ldots\right)_{n} \operatorname{Pf}\left(d_{1}, \bar{d}_{0}^{(l)}, \ldots\right)_{n} \\
& \quad-\operatorname{Pf}\left(d_{0}, d_{1}, \ldots\right)_{n} \operatorname{Pf}\left(d_{-1}, \bar{d}_{0}, \ldots\right)_{n}+\operatorname{Pf}\left(d_{0}, \bar{d}_{0}^{(l)}, \ldots\right)_{n} \operatorname{Pf}\left(d_{-1}, d_{1}, \ldots\right)_{n},
\end{aligned}
$$

together with above Pfaffian relations gives

$$
\left(\frac{\mathrm{d}}{\mathrm{d} t}+s\left(a_{l}-\bar{a}_{l}\right)\right) h_{n}^{(l)} \times f_{n}=s\left(a_{l} h_{n+1}^{(l)} f_{n-1}-\bar{a}_{l} h_{n-1}^{(l)} f_{n+1}\right)+h_{n}^{(l)}\left(\frac{\mathrm{d}}{\mathrm{d} t} f_{n}\right),
$$

which is nothing but the second bilinear equation. Now let us proceed to the proof of the third bilinear equation. To this end, we need to define

$$
\begin{aligned}
& \operatorname{Pf}\left(a_{j}, \bar{\beta}_{l}\right)=0, \quad \operatorname{Pf}\left(b_{j}, \bar{\beta}_{l}\right)= \begin{cases}\frac{0,}{\alpha_{j-N}^{(l)}}, & 1 \leq j \leq N, \\
N+1 \leq j \leq 2 N,\end{cases} \\
& \operatorname{Pf}\left(d_{0},{\overline{d_{0}^{\prime}}}^{(l)}\right)=1, \quad \operatorname{Pf}\left(\bar{c}_{j}^{(l)}, \bar{c}_{k}^{(l)}\right)_{n}=\frac{p_{j}-p_{k}}{p_{j} p_{k}-1} \frac{1-a_{l} p_{j}}{p_{j}-a_{l}} \frac{1-a_{l} p_{k}}{p_{k}-a_{l}} \varphi_{j}(n) \varphi_{k}(n), \\
& \operatorname{Pf}\left({\overline{d_{0}^{\prime}}}^{(l)}, a_{j}\right)=p_{j}^{n}\left(\frac{1-a_{l} p_{j}}{p_{j}-a_{l}}\right) e^{\eta_{j}}, \quad \operatorname{Pf}\left(\bar{c}_{j}^{(l)}, b_{k}\right)=\delta_{j k}, \quad \operatorname{Pf}\left({\overline{d_{0}^{\prime}}}^{(l)}, b_{j}\right)=0 .
\end{aligned}
$$


Then from the fact

$$
\overline{\operatorname{Pf}\left(a_{j}, a_{k}\right)}=\operatorname{Pf}\left(a_{j^{\prime}}, a_{k^{\prime}}\right), \quad \overline{\operatorname{Pf}\left(b_{j}, b_{k}\right)}=\operatorname{Pf}\left(b_{j^{\prime}}, b_{k^{\prime}}\right),
$$

where $j^{\prime}=j+N \bmod (2 N), k^{\prime}=k+N \bmod (2 N)$, we obtain

$$
\begin{aligned}
& \bar{f}_{n}=f_{n}, \quad \bar{g}_{n}^{(j)}=\operatorname{Pf}\left(d_{0}, \bar{\beta}_{j}, a_{1}, \ldots, a_{2 N}, b_{1}, \ldots, b_{2 N}\right)_{n}, \\
& \bar{h}_{n}^{(l)}=\operatorname{Pf}\left(\bar{c}_{1}^{(l)}, \ldots, \bar{c}_{2 N}^{(l)}, b_{1}, \ldots, b_{2 N}\right)=\operatorname{Pf}\left(d_{0},{\overline{d_{0}^{\prime}}}^{(l)}, \ldots\right)_{n} .
\end{aligned}
$$

Since

$$
f_{n+1}=\operatorname{Pf}\left(d_{0}, d_{1}, \ldots\right)_{n}, \quad f_{n-1}=\operatorname{Pf}\left(d_{0}, d_{-1}, \ldots\right)_{n},
$$

we then have

$$
\begin{aligned}
& f_{n+1}=f_{n}+\sum_{j=1}^{2 N}(-1)^{j-1} \operatorname{Pf}\left(d_{1}, a_{j}\right) \operatorname{Pf}\left(d_{0}, \ldots, \hat{a}_{j}, \ldots\right)_{n}, \\
& f_{n-1}=f_{n}+\sum_{j=1}^{2 N}(-1)^{j-1} \operatorname{Pf}\left(d_{-1}, a_{j}\right) \operatorname{Pf}\left(d_{0}, \ldots, \hat{a}_{j}, \ldots\right)_{n},
\end{aligned}
$$

Then we can show

$$
\begin{aligned}
f_{n+1} f_{n-1}-f_{n} f_{n}= & -\sum_{j<k}(-1)^{j+k}\left(p_{j}+\frac{1}{p_{j}}-p_{k}-\frac{1}{p_{k}}\right) \operatorname{Pf}\left(b_{j}, b_{k}\right) \\
& \times \operatorname{Pf}\left(d_{0}, \ldots, \hat{b}_{j}, \ldots\right) \operatorname{Pf}\left(d_{0}, \ldots, \hat{b}_{k}, \ldots\right) .
\end{aligned}
$$

On the other hand, since

$$
h_{n}^{(l)}=\operatorname{Pf}\left(d_{0},{\overline{d_{0}}}^{(l)}, \ldots\right)_{n}, \quad \bar{h}_{n}^{(l)}=\operatorname{Pf}\left(d_{0},{\overline{d_{0}^{\prime}}}^{(l)}, \ldots\right)_{n},
$$

we have

$$
\begin{aligned}
& h_{n}^{(l)}=f_{n}+\sum_{j=1}^{2 N}(-1)^{j-1} \operatorname{Pf}\left(d_{0}, a_{j}\right)_{n}\left(\frac{p_{j}-a_{l}}{1-a_{l} p_{j}}\right) \operatorname{Pf}\left(d_{0}, \ldots, \hat{a}_{j}, \ldots\right)_{n}, \\
& \bar{h}_{n}^{(l)}=f_{n}+\sum_{j=1}^{2 N}(-1)^{j-1} \operatorname{Pf}\left(d_{0}, a_{j}\right)_{n}\left(\frac{1-a_{l} p_{j}}{p_{j}-a_{l}}\right) \operatorname{Pf}\left(d_{0}, \ldots, \hat{a}_{j}, \ldots\right)_{n} .
\end{aligned}
$$

Similarly, we can show

$$
\begin{aligned}
\left|h_{n}^{(l)}\right|^{2}-f_{n} f_{n}= & -\sum_{j<k}(-1)^{j+k}\left(\frac{p_{j}-a_{l}}{1-a_{l} p_{j}}+\frac{1-a_{l} p_{j}}{p_{j}-a_{l}}-\frac{p_{k}-a_{l}}{1-a_{l} p_{k}}-\frac{1-a_{l} p_{k}}{p_{k}-a_{l}}\right) \\
& \times \operatorname{Pf}\left(b_{j}, b_{k}\right) \operatorname{Pf}\left(d_{0}, \ldots, \hat{b}_{j}, \ldots\right) \operatorname{Pf}\left(d_{0}, \ldots, \hat{b}_{k}, \ldots\right) .
\end{aligned}
$$

Finally, we have

$$
\begin{gathered}
s\left(f_{n+1} f_{n-1}-f_{n} f_{n}\right)-\sum_{l=1}^{M-m} \sigma_{l+m}\left|\rho_{l}\right|^{2}\left(\left|h_{n}^{(l)}\right|^{2}-f_{n} f_{n}\right) \\
=-\sum_{j<k}(-1)^{j+k}\left\{s\left(p_{j}+\frac{1}{p_{j}}-p_{k}-\frac{1}{p_{k}}\right)\right.
\end{gathered}
$$




$$
\begin{aligned}
& \left.-\sum_{l=1}^{M-m} \sigma_{l+m}\left|\rho_{l}\right|^{2}\left(\frac{p_{j}-a_{l}}{1-a_{l} p_{j}}+\frac{1-a_{l} p_{j}}{p_{j}-a_{l}}-\frac{p_{k}-a_{l}}{1-a_{l} p_{k}}-\frac{1-a_{l} p_{k}}{p_{k}-a_{l}}\right)\right\} \\
& \times \operatorname{Pf}\left(b_{j}, b_{k}\right) \operatorname{Pf}\left(d_{0}, \ldots, \hat{b}_{j}, \ldots\right) \operatorname{Pf}\left(d_{0}, \ldots, \hat{b}_{k}, \ldots\right) \\
= & \sum_{j=1}^{N} \sum_{k=N+1}^{2 N}(-1)^{j+k} \sum_{l=1}^{m} \alpha_{j}^{(l)} \sigma_{l} \overline{\alpha_{k-N}^{(l)}} \operatorname{Pf}\left(d_{0}, \ldots, \hat{b}_{j}, \ldots\right) \operatorname{Pf}\left(d_{0}, \ldots, \hat{b}_{k}, \ldots\right) \\
= & \sum_{l=1}^{m} \sigma_{l} \sum_{j=1}^{N} \sum_{k=N+1}^{2 N}(-1)^{j+k} \operatorname{Pf}\left(b_{j}, \beta_{l}\right) \operatorname{Pf}\left(b_{k}, \bar{\beta}_{l}\right) \operatorname{Pf}\left(d_{0}, \ldots, \hat{b}_{j}, \ldots\right) \operatorname{Pf}\left(d_{0}, \ldots, \hat{b}_{k}, \ldots\right) \\
= & \sum_{l=1}^{m} \sigma_{l}\left|g_{n}^{(l)}\right|^{2} .
\end{aligned}
$$

The third bilinear equation is proved.

The above Pfaffian solutions, with dependent variable transformations (2.1), give general $N$-bright-dark soliton solutions to the semi-discrete vector NLS equation (1.3).

\section{One- and two-soliton solutions for the two- and three-coupled discrete NLS equation}

\subsection{Two-component semi-discrete NLS equation}

In this subsection, we provide and illustrate one- and two-soliton for two-component semidiscrete NLS equation (1.2) explicitly.

One-soliton solution. the tau-functions for one-soliton solution $(N=1)$ are

$$
\begin{aligned}
& f_{n}=-1-c_{1 \overline{1}}\left(p_{1} \bar{p}_{1}\right)^{n} e^{\eta_{1}+\bar{\eta}_{1}}, \\
& g_{n}^{(1)}=-\alpha_{1}^{(1)} p_{1}^{n} e^{\eta_{1}}, \quad h_{n}^{(1)}=-1-d_{1 \overline{1}}^{(1)}\left(p_{1} \bar{p}_{1}\right)^{n} e^{\eta_{1}+\bar{\eta}_{1}},
\end{aligned}
$$

where

$$
c_{1 \overline{1}}=\frac{\bar{\alpha}_{1}^{(1)} \sigma_{1} \alpha_{1}^{(1)}}{\left(p_{1} \bar{p}_{1}-1\right)^{2}\left(\frac{s}{\left|p_{1}\right|^{2}}+\frac{\sigma_{2}\left|\rho_{1}\right|^{2}\left(a_{1}-\bar{a}_{1}\right)^{2}}{\left|1-a_{1} p_{1}\right|^{2}\left|1-\bar{a}_{1} p_{1}\right|^{2}}\right)}, \quad d_{1 \overline{1}}^{(1)}=-\frac{\left(p_{1}-a_{1}\right)\left(\bar{p}_{1}-a_{1}\right)}{\left(1-a_{1} p_{1}\right)\left(1-a_{1} \bar{p}_{1}\right)} c_{1 \overline{1}} .
$$

The above tau functions lead to the one-soliton solution as follows

$$
\begin{aligned}
& q_{n}^{(1)}=\frac{\alpha_{1}^{(1)}}{2 \sqrt{c_{11}}} e^{\mathrm{i} \xi_{1 I}} \operatorname{sech}\left(\xi_{1 R}+\theta_{0}\right), \\
& q_{n}^{(2)}=\frac{1}{2} \rho_{1} e^{\mathrm{i} \zeta_{1}}\left(1+e^{2 \mathrm{i} \phi_{1}}+\left(e^{2 \mathrm{i} \phi_{1}}-1\right) \tanh \left(\xi_{1 R}+\theta_{0}\right)\right),
\end{aligned}
$$

where $\xi_{1}=\xi_{1 R}+\mathrm{i} \xi_{1 I}=n \ln \left(\mathrm{i} p_{1}\right)+s\left(p_{1}-p_{1}^{-1}\right) t, \zeta_{1}=n \varphi_{1}+n \pi / 2+\omega_{1} t, e^{\mathrm{i} \varphi_{1}}=a_{1}, e^{2 \theta_{0}}=c_{1 \overline{1}}$, $e^{2 \mathrm{i} \phi_{1}}=-\left(p_{1}-a_{1}\right)\left(\bar{p}_{1}-a_{1}\right) /\left(\left(1-a_{1} p_{1}\right)\left(1-a_{1} \bar{p}_{1}\right)\right)$. Therefore, the amplitude of bright soliton for $q^{(1)}$ are $\frac{1}{2}\left|\alpha_{1}^{(1)}\right| / \sqrt{a_{11}}$. The dark soliton $q^{(2)}$ approaches $\left|\rho_{1}\right|$ as $x \rightarrow \pm \infty$. In addition, the intensity of the dark soliton is $\left|\rho_{1}\right| \cos \phi_{1}$.

An example of one-bright-dark soliton is illustrated in Fig. 1a $p_{1}=1.0+0.8 \mathrm{i}, \rho_{1}=5.0$, $\alpha_{1}^{(1)}=1.0+\mathrm{i}, a_{1}=0.8+0.6 \mathrm{i}$. for focusing-focusing case $\left(\sigma_{1}=1.0, \sigma_{2}=1.0\right)$. Fig. $1 \mathrm{~b}$ shows the bright-dark soliton for the focusing-defocusing case $\left(\sigma_{1}=1.0, \sigma_{2}=-1.0\right)$. It is interesting to note the dark soliton corresponding to defocusing component becomes an anti-dark one. 

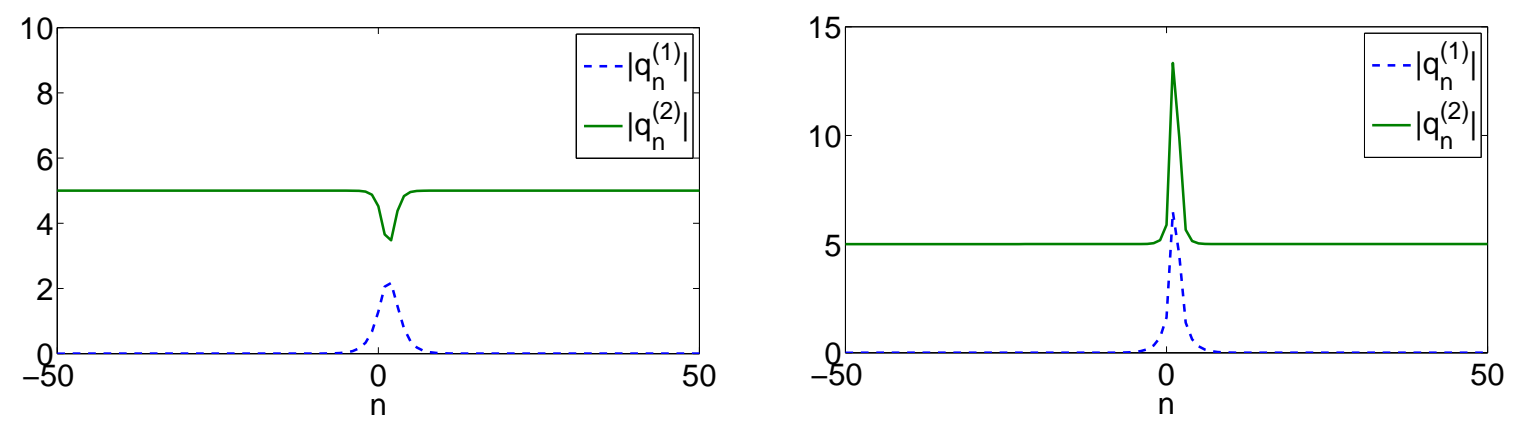

Figure 1. One-bright-dark soliton soliton solution to a two-coupled semi-discrete NLS equation: (a) focusing-focusing case $\left(\sigma_{1}=1.0, \sigma_{2}=1.0\right)$; (b) focusing-defocusing case $\left(\sigma_{1}=1.0, \sigma_{2}=-1.0\right)$.
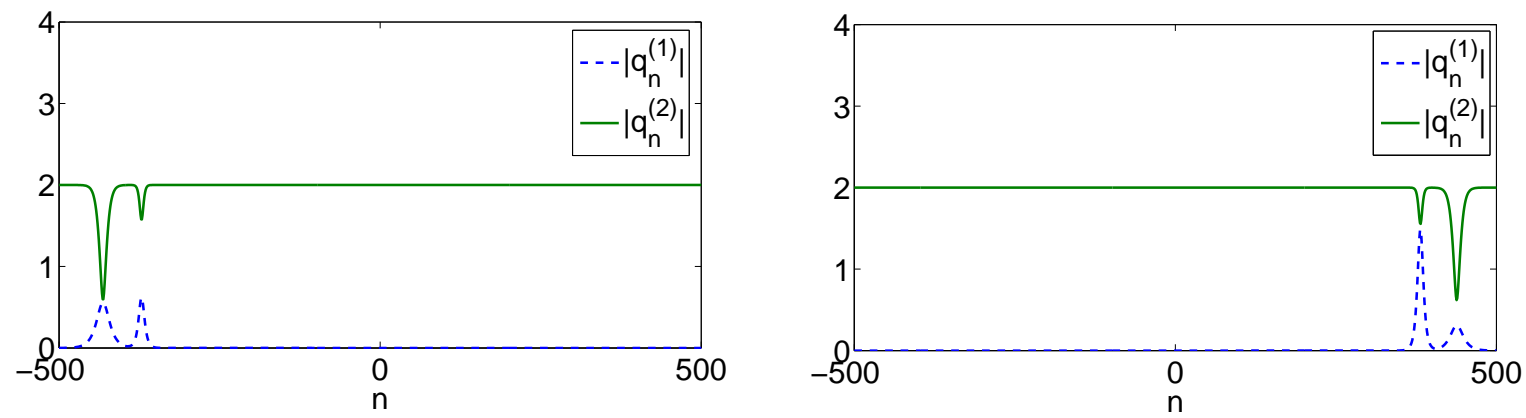

Figure 2. A two-soliton solution of mixed type for a two-coupled semi-discrete NLS equation: (a) before the collision $t=-80$; (b) after the collision $t=80$.

Two-soliton solution. The tau functions for two-soliton are of the following form

$$
\begin{aligned}
& f_{n}=1+c_{1 \overline{1}} E_{1} \bar{E}_{1}+c_{2 \overline{1}} E_{2} \bar{E}_{1}+c_{1 \overline{2}} E_{1} \bar{E}_{2}+c_{2 \overline{2}} E_{2} \bar{E}_{2}+c_{12 \overline{1} \overline{2}} E_{1} E_{2} \bar{E}_{1} \bar{E}_{2}, \\
& g_{n}=\alpha_{1}^{(1)} E_{1}+\alpha_{2}^{(1)} E_{2}+c_{12 \overline{1}}^{(j)} E_{1} E_{2} \bar{E}_{1}+c_{12 \overline{2}}^{(j)} E_{1} E_{2} \bar{E}_{2}, \\
& h_{n}=1+d_{1 \overline{1}}^{(1)} E_{1} \bar{E}_{1}+d_{2 \overline{1}}^{(1)} E_{2} \bar{E}_{1}+d_{1 \overline{2}}^{(1)} E_{1} \bar{E}_{2}+d_{2 \overline{2}}^{(1)} E_{2} \bar{E}_{2}+d_{12 \overline{1} \overline{2}}^{(1)} E_{1} E_{2} \bar{E}_{1} \bar{E}_{2},
\end{aligned}
$$

where $E_{j}=p_{j}^{n} e^{\eta_{j}}$

$$
\begin{aligned}
& c_{i \bar{j}}=\frac{\bar{\alpha}_{i}^{(1)} \sigma_{1} \alpha_{j}^{(1)}}{\left(p_{i} \bar{p}_{j}-1\right)^{2}\left(\frac{s}{p_{i} \bar{p}_{j}}+\frac{\sigma_{2}\left|\rho_{1}\right|^{2}\left(a_{1}-\bar{a}_{1}\right)^{2}}{\left|1-a_{1} p_{i}\right|^{2}\left|1-\bar{a}_{1} p_{j}\right|^{2}}\right)}, \quad d_{i \bar{j}}^{(1)}=-\frac{\left(p_{i}-a_{1}\right)\left(\bar{p}_{j}-a_{1}\right)}{\left(1-a_{1} p_{i}\right)\left(1-a_{1} \bar{p}_{j}\right)} c_{i \bar{j}}, \\
& c_{12 \overline{1} \overline{2}}=\left|p_{2}-p_{1}\right|^{2}\left(\frac{c_{1 \overline{1}} c_{2 \overline{2}}}{\left(p_{1}+\bar{p}_{2}\right)\left(p_{2}+\bar{p}_{1}\right)}-\frac{c_{1 \overline{2}} c_{2 \overline{1}}}{\left(p_{1}+\bar{p}_{1}\right)\left(p_{2}+\bar{p}_{2}\right)}\right), \\
& c_{12 \bar{j}}=\left(p_{2}-p_{1}\right)\left(\frac{\alpha_{2}^{(1)} c_{1 \bar{j}}}{p_{2}+\bar{p}_{j}}-\frac{\alpha_{1}^{(1)} c_{2 \bar{j}}}{p_{1}+\bar{p}_{j}}\right), \\
& d_{12 \overline{1} \overline{2}}=\frac{\left(p_{1}-a_{1}\right)\left(\bar{p}_{1}-a_{1}\right)\left(p_{2}-a_{2}\right)\left(\bar{p}_{1}-a_{2}\right)}{\left(1-a_{1} p_{1}\right)\left(1-a_{1} \bar{p}_{1}\right)\left(1-a_{1} p_{2}\right)\left(1-a_{1} \bar{p}_{2}\right)} c_{12 \overline{1} \overline{2} .}
\end{aligned}
$$

A two bright-dark soliton solution is shown in Fig. 2 before and after the collision for parameters $\sigma_{1}=1.0, \sigma_{2}=-1, p_{1}=1.0+0.5 \mathrm{i}, \rho=2.0, \alpha_{1}^{(1)}=1.0+0.8 \mathrm{i}, \alpha_{2}^{(1)}=1.0+0.5 \mathrm{i}, a_{1}=0.6+0.8 \mathrm{i}$. It can be seen that the collision is elastic which is the same as for the continuous two-component NLS equation. 


\subsection{Bright-dark soliton solution for three-component semi-discrete NLS equation}

In this subsection, we will give the mixed-type soliton solution to the following three-component semi-discrete NLS equation

$$
\begin{aligned}
& \mathrm{i} q_{n, t}^{(1)}=\left(1+\sum_{k=1}^{3} \sigma_{k}\left|q_{n}^{(k)}\right|^{2}\right)\left(q_{n+1}^{(1)}+q_{n-1}^{(1)}\right), \\
& \mathrm{i} q_{n, t}^{(2)}=\left(1+\sum_{k=1}^{3} \sigma_{k}\left|q_{n}^{(k)}\right|^{2}\right)\left(q_{n+1}^{(2)}+q_{n-1}^{(2)}\right), \\
& \mathrm{i} q_{n, t}^{(3)}=\left(1+\sum_{k=1}^{3} \sigma_{k}\left|q_{n}^{(k)}\right|^{2}\right)\left(q_{n+1}^{(3)}+q_{n-1}^{(3)}\right) .
\end{aligned}
$$

Two-bright-one-dark soliton solution. The tau-functions for one-soliton solution $(N=1)$ are

$$
f_{n}=-1-c_{1 \overline{1}}\left(p_{1} \bar{p}_{1}\right)^{n} e^{\eta_{1}+\bar{\eta}_{1}}, \quad g_{n}^{(j)}=-\alpha_{1}^{(j)} p_{1}^{n} e^{\eta_{1}}, \quad h_{n}^{(1)}=-1-d_{1 \overline{1}}^{(1)}\left(p_{1} \bar{p}_{1}\right)^{n} e^{\eta_{1}+\bar{\eta}_{1}},
$$

where

$$
c_{1 \overline{1}}=\frac{\sum_{k=1}^{2} \bar{\alpha}_{1}^{(k)} \sigma_{k} \alpha_{1}^{(k)}}{\left(p_{1} \bar{p}_{1}-1\right)^{2}\left(\frac{s}{\left|p_{1}\right|^{2}}+\frac{\sigma_{3}\left|\rho_{1}\right|^{2}\left(a_{1}-\bar{a}_{1}\right)^{2}}{\left|1-a_{1} p_{1}\right|^{2}\left|1-\bar{a}_{1} p_{1}\right|^{2}}\right)}, \quad d_{1 \overline{1}}^{(1)}=-\frac{\left(p_{1}-a_{1}\right)\left(\bar{p}_{1}-a_{1}\right)}{\left(1-a_{1} p_{1}\right)\left(1-a_{1} \bar{p}_{1}\right)} c_{1 \overline{1}},
$$

where $\omega=s\left(a_{1}-\bar{a}_{1}\right),\left|a_{1}\right|=1, s=1+\sigma_{3}\left|\rho_{1}\right|^{2}$ with $\bar{a}_{1}$ representing the complex conjugate of $a_{1}$. The above tau functions lead to the one-soliton as follows

$$
\begin{aligned}
& q_{n}^{(j)}=\frac{\alpha_{1}^{(j)}}{2 \sqrt{c_{1 \overline{1}}}} e^{\mathrm{i} \xi_{1 I}} \operatorname{sech}\left(\xi_{1 R}+\theta_{0}\right), \quad j=1,2 \\
& q_{n}^{(3)}=\frac{1}{2} \rho_{1} e^{\mathrm{i} \zeta_{1}}\left(1+e^{2 \mathrm{i} \phi_{1}}+\left(e^{2 \mathrm{i} \phi_{1}}-1\right) \tanh \left(\xi_{1 R}+\theta_{0}\right)\right),
\end{aligned}
$$

where $\xi_{1}=\xi_{1 R}+\mathrm{i} \xi_{1 I}=n \ln \left(\mathrm{i} p_{1}\right)+s\left(p_{1}-p_{1}^{-1}\right) t, \zeta_{1}=n \varphi_{1}+n \pi / 2+\omega_{1} t, e^{\mathrm{i} \varphi_{1}}=a_{1}, e^{2 \theta_{0}}=c_{1 \overline{1}}$, $e^{2 \mathrm{i} \phi_{1}}=-\left(p_{1}-a_{1}\right)\left(\bar{p}_{1}-a_{1}\right) /\left(\left(1-a_{1} p_{1}\right)\left(1-a_{1} \bar{p}_{1}\right)\right)$. Therefore, the amplitude of bright soliton for $q^{(j)}$ are $\frac{1}{2}\left|\alpha_{1}^{(j)}\right| / \sqrt{a_{11}}$. The dark soliton $q^{(3)}$ approaches $\left|\rho_{1}\right|$ as $x \rightarrow \pm \infty$. In addition, the intensity of the dark soliton is $\left|\rho_{1}\right| \cos \phi_{1}$.

The tau functions for two-soliton are of the following form

$$
\begin{aligned}
& f_{n}=1+c_{1 \overline{1}} E_{1} \bar{E}_{1}+c_{2 \overline{1}} E_{2} \bar{E}_{1}+c_{1 \overline{2}} E_{1} \bar{E}_{2}+c_{2 \overline{2}} E_{2} \bar{E}_{2}+c_{12 \overline{1} \overline{2}} E_{1} E_{2} \bar{E}_{1} \bar{E}_{2}, \\
& g_{n}^{(j)}=\alpha_{1}^{(j)} E_{1}+\alpha_{2}^{(j)} E_{2}+c_{12 \overline{1}}^{(j)} E_{1} E_{2} \bar{E}_{1}+c_{12 \overline{2}}^{(j)} E_{1} E_{2} \bar{E}_{2}, \quad j=1,2, \\
& h_{n}^{(1)}=1+d_{1 \overline{1}}^{(1)} E_{1} \bar{E}_{1}+d_{2 \overline{1}}^{(1)} E_{2} \bar{E}_{1}+d_{1 \overline{2}}^{(1)} E_{1} \bar{E}_{2}+d_{2 \overline{2}}^{(1)} E_{2} \bar{E}_{2}+d_{12 \overline{1} \overline{2}}^{(1)} E_{1} E_{2} \bar{E}_{1} \bar{E}_{2},
\end{aligned}
$$

where

$$
\begin{aligned}
& c_{i \bar{j}}=\frac{\sum_{k=1}^{2} \bar{\alpha}_{i}^{(k)} \sigma_{k} \alpha_{j}^{(k)}}{\left(p_{i} \bar{p}_{j}-1\right)^{2}\left(\frac{s}{p_{i} \bar{p}_{j}}+\frac{\sigma_{3}\left|\rho_{1}\right|^{2}\left(a_{1}-\bar{a}_{1}\right)^{2}}{\left|1-a_{1} p_{i}\right|^{2}\left|1-\bar{a}_{1} p_{j}\right|^{2}}\right)}, \quad d_{i \bar{j}}^{(1)}=-\frac{\left(p_{i}-a_{1}\right)\left(\bar{p}_{j}-a_{1}\right)}{\left(1-a_{1} p_{i}\right)\left(1-a_{1} \bar{p}_{j}\right)} c_{i \bar{j}}, \\
& c_{12 \overline{1} \overline{2}}=\left|p_{2}-p_{1}\right|^{2}\left(\frac{c_{1 \overline{1}} c_{2 \overline{2}}}{\left(p_{1}+\bar{p}_{2}\right)\left(p_{2}+\bar{p}_{1}\right)}-\frac{c_{1 \overline{2}} c_{2 \overline{1}}}{\left(p_{1}+\bar{p}_{1}\right)\left(p_{2}+\bar{p}_{2}\right)}\right),
\end{aligned}
$$




$$
\begin{aligned}
& c_{12 \bar{j}}=\left(p_{2}-p_{1}\right)\left(\frac{\alpha_{2}^{(1)} c_{1 \bar{j}}}{p_{2}+\bar{p}_{j}}-\frac{\alpha_{1}^{(1)} c_{2 \bar{j}}}{p_{1}+\bar{p}_{j}}\right), \\
& d_{12 \overline{1} \overline{2}}^{(1)}=\frac{\left(p_{1}-a_{1}\right)\left(\bar{p}_{1}-a_{1}\right)\left(p_{2}-a_{2}\right)\left(\bar{p}_{1}-a_{2}\right)}{\left(1-a_{1} p_{1}\right)\left(1-a_{1} \bar{p}_{1}\right)\left(1-a_{1} p_{2}\right)\left(1-a_{1} \bar{p}_{2}\right)} c_{12 \overline{1} \overline{2}} .
\end{aligned}
$$

It is found that two-bright-one-dark soliton solution given above is nonsingular for any other combinations of nonlinearities if the following quantity

$$
\left(\sum_{k=1}^{2} \bar{\alpha}_{i}^{(k)} \sigma_{k} \alpha_{j}^{(k)}\right)\left(\frac{s}{\left|p_{i}\right|^{2}}+\frac{\sigma_{3}\left|\rho_{1}\right|^{2}\left(a_{1}-\bar{a}_{1}\right)^{2}}{\left|1-a_{1} p_{i}\right|^{2}\left|1-\bar{a}_{1} p_{i}\right|^{2}}\right)
$$

is positive.

One-bright-two-dark soliton solution. The tau-functions for one-soliton solution $(N=1)$ are

$$
f_{n}=-1-c_{1 \overline{1}}\left(p_{1} \bar{p}_{1}\right)^{n} e^{\eta_{1}+\bar{\eta}_{1}}, \quad g_{n}^{(1)}=-\alpha_{1}^{(1)} p_{1}{ }^{n} e^{\eta_{1}}, \quad h_{n}^{(l)}=-1-d_{1 \overline{1}}^{(l)}\left(p_{1} \bar{p}_{1}\right)^{n} e^{\eta_{1}+\bar{\eta}_{1}},
$$

where

$$
c_{1 \overline{1}}=\frac{\bar{\alpha}_{1}^{(1)} \sigma_{1} \alpha_{1}^{(1)}}{\left(p_{1} \bar{p}_{1}-1\right)^{2}\left(\frac{s}{\left|p_{1}\right|^{2}}+\frac{\sum_{l=1}^{2} \sigma_{l+1}\left|\rho_{l}\right|^{2}\left(a_{l}-\bar{a}_{l}\right)^{2}}{\left|1-a_{1} p_{1}\right|^{2}\left|1-\bar{a}_{1} p_{1}\right|^{2}}\right)} .
$$

Here $\omega_{l}=s\left(a_{l}-\bar{a}_{l}\right),\left|a_{l}\right|=1, s=1+\sigma_{2}\left|\rho_{1}\right|^{2}+\sigma_{3}\left|\rho_{2}\right|^{2}, \bar{a}_{l}$ represents the complex conjugate of $a_{l}$. The above tau functions lead to the one-soliton as follows

$$
\begin{aligned}
& q_{n}^{(1)}=\frac{\alpha_{1}^{(1)}}{2} \sqrt{c_{1 \overline{1}}} e^{\mathrm{i} \xi_{1 I}} \operatorname{sech}\left(\xi_{1 R}+\theta_{0}\right), \\
& q_{n}^{(l+1)}=\frac{1}{2} \rho_{l} e^{\mathrm{i} \zeta_{l}}\left(1+e^{2 \mathrm{i} \phi_{l}}+\left(e^{2 \mathrm{i} \phi_{l}}-1\right) \tanh \left(\xi_{1 R}+\theta_{0}\right)\right), \quad l=1,2,
\end{aligned}
$$

where $\xi_{1}=\xi_{1 R}+\mathrm{i} \xi_{1 I}=n \ln \left(\mathrm{i} p_{1}\right)+s\left(p_{1}-p_{1}^{-1}\right) t, \zeta_{l}=n \varphi_{l}+n \pi / 2+\omega_{l} t, e^{\mathrm{i} \varphi_{l}}=a_{l}, e^{2 \theta_{0}}=c_{1 \overline{1}}$, $e^{2 \mathrm{i} \phi_{l}}=-\left(p_{1}-a_{l}\right)\left(\bar{p}_{1}-a_{l}\right) /\left(\left(1-a_{l} p_{1}\right)\left(1-a_{l} \bar{p}_{1}\right)\right)$. Therefore, the amplitude of bright soliton for $q^{(1)}$ are $\frac{1}{2}\left|\alpha_{1}^{(1)}\right| / \sqrt{a_{11}}$. The dark soliton $q^{(l+1)}$ approaches $\left|\rho_{l}\right|$ as $x \rightarrow \pm \infty$. In addition, the intensity of the dark soliton is $\left|\rho_{l}\right| \cos \phi_{l}$.

The tau functions for two-soliton are of the following form

$$
\begin{aligned}
& f_{n}=1+c_{1 \overline{1}} E_{1} \bar{E}_{1}+c_{2 \overline{1}} E_{2} \bar{E}_{1}+c_{1 \overline{2}} E_{1} \bar{E}_{2}+c_{2 \overline{2}} E_{2} \bar{E}_{2}+c_{12 \overline{1} \overline{2}} E_{1} E_{2} \bar{E}_{1} \bar{E}_{2}, \\
& g_{n}^{(1)}=\alpha_{1}^{(1)} E_{1}+\alpha_{2}^{(1)} E_{2}+c_{12 \overline{1}}^{(1)} E_{1} E_{2} \bar{E}_{1}+c_{12 \overline{2}}^{(1)} E_{1} E_{2} \bar{E}_{2}, \\
& h_{n}^{(l)}=1+d_{1 \overline{1}}^{(l)} E_{1} \bar{E}_{1}+d_{2 \overline{1}}^{(l)} E_{2} \bar{E}_{1}+d_{1 \overline{2}}^{(l)} E_{1} \bar{E}_{2}+d_{2 \overline{2}}^{(l)} E_{2} \bar{E}_{2}+d_{12 \overline{1} \overline{2}}^{(l)} E_{1} E_{2} \bar{E}_{1} \bar{E}_{2}, \quad l=1,2,
\end{aligned}
$$

where

$$
\begin{aligned}
& c_{i \bar{j}}=\frac{\bar{\alpha}_{i}^{(1)} \sigma_{1} \alpha_{j}^{(1)}}{\left(p_{i} \bar{p}_{j}-1\right)^{2}\left(\frac{s}{p_{i} \bar{p}_{j}}+\frac{\sum_{l=1}^{2} \sigma_{l+1}\left|\rho_{l}\right|^{2}\left(a_{l}-\bar{a}_{l}\right)^{2}}{\left|1-a_{l} p_{i}\right|^{2}\left|1-\bar{a}_{l} p_{j}\right|^{2}}\right)}, \quad d_{i \bar{j}}^{(l)}=-\frac{\left(p_{i}-a_{l}\right)\left(\bar{p}_{j}-a_{l}\right)}{\left(1-a_{l} p_{i}\right)\left(1-a_{l} \bar{p}_{j}\right)} c_{i \bar{j}}, \\
& c_{12 \overline{1} \overline{2}}=\left|p_{2}-p_{1}\right|^{2}\left(\frac{c_{1 \overline{1}} c_{2 \overline{2}}}{\left(p_{1}+\bar{p}_{2}\right)\left(p_{2}+\bar{p}_{1}\right)}-\frac{c_{1 \overline{2}} c_{2 \overline{1}}}{\left(p_{1}+\bar{p}_{1}\right)\left(p_{2}+\bar{p}_{2}\right)}\right),
\end{aligned}
$$




$$
\begin{aligned}
& c_{12 \bar{j}}=\left(p_{2}-p_{1}\right)\left(\frac{\alpha_{2}^{(1)} c_{1 \bar{j}}}{p_{2}+\bar{p}_{j}}-\frac{\alpha_{1}^{(1)} c_{2 \bar{j}}}{p_{1}+\bar{p}_{j}}\right), \\
& d_{12 \overline{1} \overline{2}}^{(l)}=\frac{\left(p_{1}-a_{l}\right)\left(\bar{p}_{1}-a_{l}\right)\left(p_{2}-a_{l}\right)\left(\bar{p}_{1}-a_{l}\right)}{\left(1-a_{l} p_{1}\right)\left(1-a_{l} \bar{p}_{1}\right)\left(1-a_{l} p_{2}\right)\left(1-a_{l} \bar{p}_{2}\right)} c_{12 \overline{1} \overline{2}} .
\end{aligned}
$$

For all possible combinations of mixed type in three-coupled NLS equation, one-bright-twodark soliton solution exists if the following quantity

$$
\left(\bar{\alpha}_{i}^{(1)} \sigma_{1} \alpha_{i}^{(1)}\right)\left(\frac{s}{\left|p_{i}\right|^{2}}+\frac{\sum_{l=1}^{2} \sigma_{l+1}\left|\rho_{l}\right|^{2}\left(a_{l}-\bar{a}_{l}\right)^{2}}{\left|1-a_{l} p_{i}\right|^{2}\left|1-\bar{a}_{l} p_{i}\right|^{2}}\right)
$$

is positive.

The asymptotic analysis for two-soliton solution is performed in Appendix A. It should be pointed out that two-soliton for one-bright-two-dark soliton case always undertakes elastic collision without shape changing.

\section{Discussion and conclusion}

We conclude the present paper by two comments. First, we comment on a connection of the vector semi-discrete NLS equation to the vector modified Volterra lattice equation studied in [7]. To this end, we consider the two-component semi-discrete NLS equation of focusing type

$$
\begin{aligned}
& \mathrm{i} \frac{\mathrm{d}}{\mathrm{d} t} u_{n}=\left(1+\left|u_{n}\right|^{2}+\left|v_{n}\right|^{2}\right)\left(u_{n+1}+u_{n-1}\right), \\
& \mathrm{i} \frac{\mathrm{d}}{\mathrm{d} t} v_{n}=\left(1+\left|u_{n}\right|^{2}+\left|v_{n}\right|^{2}\right)\left(v_{n+1}+v_{n-1}\right) .
\end{aligned}
$$

Let $u_{n}=x_{n}+\mathrm{i} y_{n}$ and $v_{n}=z_{n}+\mathrm{i} w_{n}$, then equation (4.1) becomes

$$
\begin{aligned}
& \frac{\mathrm{d}}{\mathrm{d} t} x_{n}=\left(1+x_{n}^{2}+y_{n}^{2}+z_{n}^{2}+w_{n}^{2}\right)\left(y_{n+1}+y_{n-1}\right), \\
& -\frac{\mathrm{d}}{\mathrm{d} t} y_{n}=\left(1+x_{n}^{2}+y_{n}^{2}+z_{n}^{2}+w_{n}^{2}\right)\left(x_{n+1}+x_{n-1}\right), \\
& \frac{\mathrm{d}}{\mathrm{d} t} z_{n}=\left(1+x_{n}^{2}+y_{n}^{2}+z_{n}^{2}+w_{n}^{2}\right)\left(w_{n+1}+w_{n-1}\right), \\
& -\frac{\mathrm{d}}{\mathrm{d} t} w_{n}=\left(1+x_{n}^{2}+y_{n}^{2}+z_{n}^{2}+w_{n}^{2}\right)\left(z_{n+1}+z_{n-1}\right) .
\end{aligned}
$$

By defining

$$
\begin{aligned}
U_{n}^{(1)} & =\left\{\begin{array}{ll}
x_{n}, & \text { for } n \text { even, } \\
y_{n}, & \text { for } n \text { odd },
\end{array} \quad U_{n}^{(2)}= \begin{cases}-y_{n}, & \text { for } n \text { even, } \\
x_{n}, & \text { for } n \text { odd },\end{cases} \right. \\
U_{n}^{(3)} & =\left\{\begin{array}{ll}
z_{n}, & \text { for } n \text { even, } \\
w_{n}, & \text { for } n \text { odd },
\end{array} \quad U_{n}^{(4)}= \begin{cases}-w_{n}, & \text { for } n \text { even, } \\
z_{n}, & \text { for } n \text { odd },\end{cases} \right. \\
U_{n}^{(5)} & = \begin{cases}(-1)^{n / 2}, & \text { for } n \text { even, } \\
(-1)^{(n-1) / 2}, & \text { for } n \text { odd },\end{cases}
\end{aligned}
$$

we obtain

$$
\frac{\mathrm{d}}{\mathrm{d} t} U_{n}^{(1)}=\left(\left(U_{n}^{(1)}\right)^{2}+\left(U_{n}^{(2)}\right)^{2}+\left(U_{n}^{(3)}\right)^{2}+\left(U_{n}^{(4)}\right)^{2}+\left(U_{n}^{(5)}\right)^{2}\right)\left(U_{n+1}^{(1)}+U_{n-1}^{(1)}\right),
$$




$$
\begin{aligned}
& \frac{\mathrm{d}}{\mathrm{d} t} U_{n}^{(2)}=\left(\left(U_{n}^{(1)}\right)^{2}+\left(U_{n}^{(2)}\right)^{2}+\left(U_{n}^{(3)}\right)^{2}+\left(U_{n}^{(4)}\right)^{2}+\left(U_{n}^{(5)}\right)^{2}\right)\left(U_{n+1}^{(2)}+U_{n-1}^{(2)}\right), \\
& \frac{\mathrm{d}}{\mathrm{d} t} U_{n}^{(3)}=\left(\left(U_{n}^{(1)}\right)^{2}+\left(U_{n}^{(2)}\right)^{2}+\left(U_{n}^{(3)}\right)^{2}+\left(U_{n}^{(4)}\right)^{2}+\left(U_{n}^{(5)}\right)^{2}\right)\left(U_{n+1}^{(3)}+U_{n-1}^{(3)}\right), \\
& \frac{\mathrm{d}}{\mathrm{d} t} U_{n}^{(4)}=\left(\left(U_{n}^{(1)}\right)^{2}+\left(U_{n}^{(2)}\right)^{2}+\left(U_{n}^{(3)}\right)^{2}+\left(U_{n}^{(4)}\right)^{2}+\left(U_{n}^{(5)}\right)^{2}\right)\left(U_{n+1}^{(4)}+U_{n-1}^{(4)}\right), \\
& \frac{\mathrm{d}}{\mathrm{d} t} U_{n}^{(5)}=\left(\left(U_{n}^{(1)}\right)^{2}+\left(U_{n}^{(2)}\right)^{2}+\left(U_{n}^{(3)}\right)^{2}+\left(U_{n}^{(4)}\right)^{2}+\left(U_{n}^{(5)}\right)^{2}\right)\left(U_{n+1}^{(5)}+U_{n-1}^{(5)}\right)
\end{aligned}
$$

for $n$ being even, and

$$
\begin{aligned}
& \frac{\mathrm{d}}{\mathrm{d} t} U_{n}^{(1)}=-\left(\left(U_{n}^{(1)}\right)^{2}+\left(U_{n}^{(2)}\right)^{2}+\left(U_{n}^{(3)}\right)^{2}+\left(U_{n}^{(4)}\right)^{2}+\left(U_{n}^{(5)}\right)^{2}\right)\left(U_{n+1}^{(1)}+U_{n-1}^{(1)}\right), \\
& \frac{\mathrm{d}}{\mathrm{d} t} U_{n}^{(2)}=-\left(\left(U_{n}^{(1)}\right)^{2}+\left(U_{n}^{(2)}\right)^{2}+\left(U_{n}^{(3)}\right)^{2}+\left(U_{n}^{(4)}\right)^{2}+\left(U_{n}^{(5)}\right)^{2}\right)\left(U_{n+1}^{(2)}+U_{n-1}^{(2)}\right), \\
& \frac{\mathrm{d}}{\mathrm{d} t} U_{n}^{(3)}=-\left(\left(U_{n}^{(1)}\right)^{2}+\left(U_{n}^{(2)}\right)^{2}+\left(U_{n}^{(3)}\right)^{2}+\left(U_{n}^{(4)}\right)^{2}+\left(U_{n}^{(5)}\right)^{2}\right)\left(U_{n+1}^{(3)}+U_{n-1}^{(3)}\right), \\
& \frac{\mathrm{d}}{\mathrm{d} t} U_{n}^{(4)}=-\left(\left(U_{n}^{(1)}\right)^{2}+\left(U_{n}^{(2)}\right)^{2}+\left(U_{n}^{(3)}\right)^{2}+\left(U_{n}^{(4)}\right)^{2}+\left(U_{n}^{(5)}\right)^{2}\right)\left(U_{n+1}^{(4)}+U_{n-1}^{(4)}\right), \\
& \frac{\mathrm{d}}{\mathrm{d} t} U_{n}^{(5)}=-\left(\left(U_{n}^{(1)}\right)^{2}+\left(U_{n}^{(2)}\right)^{2}+\left(U_{n}^{(3)}\right)^{2}+\left(U_{n}^{(4)}\right)^{2}+\left(U_{n}^{(5)}\right)^{2}\right)\left(U_{n+1}^{(5)}+U_{n-1}^{(5)}\right)
\end{aligned}
$$

for $n$ being odd, in other words,

$$
\frac{\mathrm{d}}{\mathrm{d} t} U_{n}^{(j)}=(-1)^{n}\left(\sum_{k=1}^{5}\left(U_{n}^{(k)}\right)^{2}\right)\left(U_{n+1}^{(j)}+U_{n-1}^{(j)}\right)
$$

for $1 \leq j \leq 5$. By rewriting $U_{n}^{(j)}=\mathrm{i}^{n} V_{n}^{(j)}$ and $t=x / \mathrm{i}$, we have

$$
\frac{\mathrm{d}}{\mathrm{d} x} V_{n}^{(j)}=\left(\sum_{k=1}^{5}\left(V_{n}^{(k)}\right)^{2}\right)\left(V_{n+1}^{(j)}-V_{n-1}^{(j)}\right) .
$$

Consequently,

$$
\begin{aligned}
& u_{n}=\left\{\begin{array}{ll}
U_{n}^{(1)}-\mathrm{i} U_{n}^{(2)}, & n \text { even, } \\
U_{n}^{(2)}+\mathrm{i} U_{n}^{(1)}, & n \text { odd }
\end{array}= \begin{cases}\mathrm{i}^{n-1}\left(V_{n}^{(2)}+\mathrm{i} V_{n}^{(1)}\right), & n \text { even, } \\
\mathrm{i}^{n}\left(V_{n}^{(2)}+\mathrm{i} V_{n}^{(1)}\right), & n \text { odd },\end{cases} \right. \\
& v_{n}=\left\{\begin{array}{ll}
U_{n}^{(3)}-\mathrm{i} U_{n}^{(4)}, & n \text { even, } \\
U_{n}^{(4)}+\mathrm{i} U_{n}^{(3)}, & n \text { odd }
\end{array}= \begin{cases}\mathrm{i}^{n-1}\left(V_{n}^{(4)}+\mathrm{i} V_{n}^{(3)}\right), & n \text { even, } \\
\mathrm{i}^{n}\left(V_{n}^{(4)}+\mathrm{i} V_{n}^{(3)}\right), & n \text { odd } .\end{cases} \right.
\end{aligned}
$$

$u_{n}$ and $u_{n}^{*}$ correspond to $U_{n}^{(1)}$ and $U_{n}^{(2)}$, and $v_{n}$ and $v_{n}^{*}$ correspond to $U_{n}^{(3)}$ and $U_{n}^{(4)}$, with even-odd parity depending gauge factor $\mathrm{i}^{(n \text { or } n-1)}$.

The correspondence between coupled defocusing-defocusing and focusing-defocusing coupled Ablowitz-Ladik equation and the vector modified Volterra lattice equatino can be constructed by similar variable transformations. In all cases, we need 5 -components in the vector modified Volterra lattice, one of which is a trivial wave $V_{n}^{(5)}=\mathrm{i}^{(0 \text { or } 1)}$, in order to recover 2-component coupled Ablowitz-Ladik equation. In principle any solutions of coupled Ablowitz-Ladik equation can be derived from those of vector modified Volterra lattice and vice versa. However it is not so easy to make exact matching.

Second, we give a comparison between the NLS-type equations and the sine/sinh-Gordontype equations since their belong to the simplest positive and negative flows of the AKNS hierarchy, respectively. In a series papers by Barashenlov, Getmanov et al. [9, 10, 11, 12], 
a generic integrable relativistic system associated with the $\mathfrak{s l}(2, \mathbb{C})$ was systematically investigated, from which the massive Thirring model, the complex sine-Gordon equation in Euclidean and Minkowski spaces etc. are produced by different reductions. Especially an $\mathrm{O}(1,1)$ sineGordon equation with the Lagrangian

$$
L=\frac{u_{1 \xi} u_{1 \eta}-u_{2 \xi} u_{2 \eta}}{1-\left(u_{1}^{2}-u_{2}^{2}\right)}+\left(u_{1}^{2}-u_{2}^{2}-1\right),
$$

exhibits nontrivial interaction of solitons such as decay and fusion [10]. Here $u_{1}$ and $u_{2}$ are real variables. In parallel to the $\mathrm{O}(1,1)$ sine-Gordon equation, we can have a NLS equation of $\mathrm{O}(1,1)$ type, whose Lagrangian is

$$
L=u_{1} u_{2 t}-u_{1 t} u_{2}+\left(u_{1 x}^{2}-u_{2 x}^{2}\right)-\sigma\left(u_{1}^{2}-u_{2}^{2}\right)^{2} .
$$

On the contrary, in parallel to the U(1,1) coupled NLS system, e.g., system (1.1) with $\sigma=1$ and $\sigma=-1$, whose Lagrangian can be written by

$$
L=\frac{\mathrm{i}}{2}\left(u_{t} u^{*}-u^{*} u_{t}-v_{t} v^{*}+v^{*} v_{t}\right)+\left(|u|_{x}^{2}-|v|_{x}^{2}\right)-\sigma\left(|u|^{2}-|v|^{2}\right)^{2},
$$

where $u$ and $v$ are complex variable and ${ }^{*}$ represents complex conjugate, we could propose a $\mathrm{U}(1,1)$ coupled complex sine-Gordon system with Lagrangian

$$
L=\frac{u_{\eta} u_{\xi}^{*}-v_{\eta} v_{\xi}^{*}}{1-\left(|u|^{2}-|v|^{2}\right)}-\left(|u|^{2}-|v|^{2}\right) .
$$

Then several natural questions arise: does the NLS equation of $\mathrm{O}(1,1)$ type exhibit nontrivial interaction of solitons such as decay and fusion? Are there any nontrivial interaction of solitons for the $\mathrm{U}(1,1)$ coupled NLS system and the $\mathrm{U}(1,1)$ complex sine-Gordon system? Unfortunately, the answers to these questions are not clear at this moment. We expect above questions could be answered by the authors or others in the near future.

\section{A Appendix}

By taking $N=1$ we get the tau functions for one-soliton solution,

$$
\begin{aligned}
& f_{n}=\operatorname{Pf}\left(a_{1}, a_{2}, b_{1}, b_{2}\right)=-1-c_{1 \overline{1}} E_{1} \bar{E}_{1}, \\
& g_{n}^{(j)}=\operatorname{Pf}\left(d_{0}, \beta_{j}, a_{1}, a_{2}, b_{1}, b_{2}\right)=-\alpha_{1}^{(1)} E_{1}, \quad j=1, \ldots, m, \\
& h_{n}^{(l)}=\operatorname{Pf}\left(c_{1}^{(l)}, c_{2}^{(l)}, b_{1}, b_{2}\right)=-1-d_{1 \overline{1}}^{(l)} E_{1} \bar{E}_{1}, \quad l=1, \ldots, M-m,
\end{aligned}
$$

where

$$
c_{1 \overline{1}}=\frac{\sum_{k=1}^{m} \bar{\alpha}_{1}^{(k)} \sigma_{k} \alpha_{1}^{(k)}}{\left(p_{1} \bar{p}_{1}-1\right)^{2}\left(\frac{s}{\left|p_{1}\right|^{2}}+\sum_{l=1}^{M-m} \frac{\left|\rho_{l}\right|^{2}\left(a_{l}-\bar{a}_{l}\right)^{2}}{\left|1-a_{l} p_{1}\right|^{2}\left|1-\bar{a}_{l} p_{1}\right|^{2}}\right)}, \quad d_{1 \overline{1}}^{(l)}=-\frac{\left(p_{1}-a_{l}\right)\left(\bar{p}_{1}-a_{l}\right)}{\left(1-a_{l} p_{1}\right)\left(1-a_{l} \bar{p}_{1}\right)} c_{1 \overline{1}} .
$$

Based on the $N$-soliton solution of the vector discrete NLS equation, the tau-functions for two-soliton solution can be expanded for $N=2$

$$
\begin{aligned}
f & =\operatorname{Pf}\left(a_{1}, a_{2}, a_{3}, a_{4}, b_{1}, b_{2}, b_{3}, b_{4}\right) \\
& =1+c_{1 \overline{1}} E_{1} \bar{E}_{1}+c_{2 \overline{1}} E_{2} \bar{E}_{1}+c_{1 \overline{2}} E_{1} \bar{E}_{2}+c_{2} \overline{2} E_{2} \bar{E}_{2}+c_{12 \overline{1} \overline{2}} E_{1} E_{2} \bar{E}_{1} \bar{E}_{2},
\end{aligned}
$$




$$
\begin{aligned}
g_{n}^{(j)}= & \operatorname{Pf}\left(d_{0}, \beta_{j}, a_{1}, a_{2}, a_{3}, a_{4}, b_{1}, b_{2}, b_{3}, b_{4}\right) \\
= & \alpha_{1}^{(j)} E_{1}+\alpha_{2}^{(j)} E_{2}+c_{12 \overline{1}}^{(j)} E_{1} E_{2} \bar{E}_{1}+c_{12 \overline{2}}^{(j)} E_{1} E_{2} \bar{E}_{2}, \quad j=1, \ldots, m, \\
h_{n}^{(l)}= & \operatorname{Pf}\left(c_{1}^{(l)}, c_{2}^{(l)}, c_{3}^{(l)}, c_{4}^{(l)}, b_{1}, b_{2}, b_{3}, b_{4}\right)=1+d_{1 \overline{1}}^{(l)} E_{1} \bar{E}_{1} \\
& +d_{2 \overline{1}}^{(l)} E_{2} \bar{E}_{1}+d_{1 \overline{2}}^{(l)} E_{1} \bar{E}_{2}+d_{2 \overline{2}}^{(l)} E_{2} \bar{E}_{2}+d_{12 \overline{1} \overline{2}}^{(l)} E_{1} E_{2} \bar{E}_{1} \bar{E}_{2}, \quad l=1, \ldots, M-m,
\end{aligned}
$$

where

$$
\begin{aligned}
& c_{i \bar{j}}=\frac{\sum_{k=1}^{m} \bar{\alpha}_{j}^{(k)} \sigma_{k} \alpha_{i}^{(k)}}{\left(p_{i} \bar{p}_{j}-1\right)^{2}\left(\frac{s}{p_{i} \bar{p}_{j}}+\sum_{l=1}^{M-m} \frac{\left.\rho_{l}\right|^{2}\left(a_{l}-\bar{a}_{l}\right)^{2}}{\left|1-a_{l} p_{i}\right|^{2}\left|1-\bar{a}_{l} p_{j}\right|^{2}}\right)}, \quad d_{i \bar{j}}^{(l)}=-\frac{\left(p_{i}-a_{l}\right)\left(\bar{p}_{j}-a_{l}\right)}{\left(1-a_{l} p_{i}\right)\left(1-a_{l} \bar{p}_{j}\right)} c_{i \bar{j}}, \\
& c_{12 \overline{1} \overline{2}}=\left|P_{12}\right|^{2}\left(P_{1 \overline{1}} P_{2 \overline{2}} c_{1 \overline{2}} c_{2 \overline{1}}-P_{1 \overline{2}} P_{2 \overline{1}} c_{1 \overline{1}} c_{2 \overline{2}}\right), \quad c_{12 \bar{j}}^{(k)}=P_{12}\left(\alpha_{1}^{(k)} P_{1 \overline{1}} c_{2 \bar{j}}-\alpha_{2}^{(k)} P_{2 \overline{1}} c_{1 \bar{j}}\right), \\
& d_{12 \overline{1} \overline{2}}^{(l)}=\frac{\left(p_{1}-a_{l}\right)\left(\bar{p}_{1}-a_{l}\right)\left(p_{2}-a_{l}\right)\left(\bar{p}_{1}-a_{l}\right)}{\left(1-a_{l} p_{1}\right)\left(1-a_{l} \bar{p}_{1}\right)\left(1-a_{l} p_{2}\right)\left(1-a_{l} \bar{p}_{2}\right)} c_{12 \overline{1} \overline{2} \overline{2}}, \\
& P_{i j}=\frac{p_{i}-p_{j}}{p_{i} p_{j}-1}, \quad P_{i \bar{j}}=\frac{p_{i}-\bar{p}_{j}}{p_{i} \bar{p}_{j}-1} .
\end{aligned}
$$

Next, we investigate the asymptotic behavior of two-soliton solution. To this end, we assume $\left(\operatorname{Re} \ln \left(\mathrm{i} p_{2}\right)\right)>\operatorname{Re}\left(\ln \left(\mathrm{i} p_{1}\right)\right)>0, s \operatorname{Re}\left(p_{2}-p_{2}^{-1}\right) / \operatorname{Re}\left(\ln \left(\mathrm{i} p_{2}\right)\right)>s \operatorname{Re}\left(p_{1}-p_{1}^{-1}\right) / \operatorname{Re}\left(\ln \left(\mathrm{i} p_{1}\right)\right)$ without loss of generality. For the above choice of parameters, we have (i) $\eta_{1 R} \approx 0, \eta_{2 R} \rightarrow \mp \infty$ as $t \rightarrow \mp \infty$ for soliton 1 and (ii) $\eta_{2 R} \approx 0, \eta_{1 R} \rightarrow \pm \infty$ as $t \rightarrow \mp \infty$ for soliton 2 . This leads to the following asymptotic forms for two-soliton solution.

(i) Before collision $(t \rightarrow-\infty)$. Soliton $1\left(\eta_{1 R} \approx 0, \eta_{2 R} \rightarrow-\infty\right)$ :

$$
\begin{aligned}
q_{n}^{(j)} \rightarrow & \alpha_{1}^{(j)} \frac{\mathrm{i}^{n} E_{1}}{1+c_{1 \overline{1}} E_{1} \bar{E}_{1}} \rightarrow A_{j}^{1-} e^{\mathrm{i} \xi_{1 I}} \operatorname{sech}\left(\xi_{1 R}+\xi_{0}^{1-}\right), \\
q_{n}^{(l+m)} & \rightarrow \frac{1+d_{1 \overline{1}}^{(l)} E_{1} \bar{E}_{1}}{1+c_{1 \overline{1}} E_{1} \bar{E}_{1}} \rho_{l}\left(\mathrm{i} a_{l}\right)^{n} e^{\omega_{l} t} \\
& \rightarrow \frac{1}{2} \rho_{l} e^{\mathrm{i} \zeta_{l}}\left(1+e^{2 \mathrm{i} \phi_{l}^{(1)}}+\left(e^{2 \mathrm{i} \phi_{l}^{(1)}}-1\right) \tanh \left(\xi_{1 R}+\xi_{0}^{1-}\right)\right),
\end{aligned}
$$

where

$$
\begin{aligned}
& A_{j}^{1-}=\frac{\alpha_{1}^{(j)}}{2 \sqrt{c_{1}}}, \quad e^{2 \xi_{0}^{1-}}=c_{1 \overline{1}}, \\
& e^{2 \mathrm{i} \phi_{l}^{(1)}}=-\frac{\left(p_{1}-a_{l}\right)\left(\bar{p}_{1}-a_{l}\right)}{\left(1-a_{l} p_{1}\right)\left(1-a_{l} \bar{p}_{1}\right)}, \quad \zeta_{l}=n \varphi_{l}+n \pi / 2+\omega_{l} t \\
& \xi_{1}=\xi_{1 R}+\mathrm{i} \xi_{1 I}=n \ln \left(\mathrm{i} p_{1}\right)+s\left(p_{1}-p_{1}^{-1}\right) t, \quad e^{\mathrm{i} \varphi_{l}}=a_{l} .
\end{aligned}
$$

Soliton $2\left(\eta_{2 R} \approx 0, \eta_{1 R} \rightarrow \infty\right)$ :

$$
\begin{aligned}
q_{n}^{(j)} \rightarrow & \frac{\mathrm{i}^{n} c_{12 \overline{1}}^{(j)} E_{2}}{c_{1 \overline{1}}+c_{12 \overline{1} \overline{2}} E_{2} \bar{E}_{2}} \rightarrow A_{j}^{2-} e^{\mathrm{i} \xi_{2 I}} \operatorname{sech}\left(\xi_{2 R}+\xi_{0}^{2-}\right), \\
q_{n}^{(l+m)} & \rightarrow \frac{d_{1 \overline{1}}^{(l)}+d_{12 \overline{1} \overline{2} E_{2} \bar{E}_{2}}^{(l)} \rho_{l}\left(\mathrm{i} a_{l}\right)^{n} e^{\omega_{l} t}}{c_{1 \overline{1}}+c_{12 \overline{1} \overline{2} E_{2} \bar{E}_{2}}} \\
& \rightarrow \frac{1}{2} \rho_{l} e^{\mathrm{i}\left(\zeta_{l}+2 \phi_{l}^{(1)}\right)}\left(1+e^{2 \mathrm{i} \phi_{l}^{(2)}}+\left(e^{2 \mathrm{i} \phi_{l}^{(2)}}-1\right) \tanh \left(\xi_{2 R}+\xi_{0}^{2-}\right)\right),
\end{aligned}
$$


where

$$
\begin{aligned}
& A_{j}^{2-}=\frac{c_{12 \overline{1}}^{(j)}}{2 \sqrt{c_{1} \overline{1}} \sqrt{c_{12 \overline{1} \overline{2}}}}, \quad e^{2 \xi_{0}^{2-}}=\frac{c_{12 \overline{1} \overline{2}}}{c_{1 \overline{1}}}, \\
& e^{2 \mathrm{i} \phi_{l}^{(2)}}=-\frac{\left(p_{2}-a_{l}\right)\left(\bar{p}_{2}-a_{l}\right)}{\left(1-a_{l} p_{2}\right)\left(1-a_{l} \bar{p}_{2}\right)}, \quad \zeta_{0}^{2-}=n \varphi_{l}+n \pi / 2+\omega_{l} t \\
& \xi_{2}=\xi_{2 R}+\mathrm{i} \xi_{2 I}=n \ln \left(\mathrm{i} p_{2}\right)+s\left(p_{2}-p_{2}^{-1}\right) t .
\end{aligned}
$$

(ii) After the collision $(t \rightarrow \infty)$. Soliton $1\left(\eta_{1 R} \approx 0, \eta_{2 R} \rightarrow+\infty\right)$ :

$$
\begin{aligned}
q_{n}^{(j)} \rightarrow & \frac{c_{12 \overline{2}}^{(j)} \mathrm{i}^{n} E_{1}}{c_{2} \overline{2}+c_{12 \overline{1} \overline{2}} E_{1} \bar{E}_{1}} \rightarrow A_{j}^{1+} e^{\mathrm{i} \xi_{1 I}} \operatorname{sech}\left(\xi_{1 R}+\xi_{0}^{1+}\right), \\
q_{n}^{(l+m)} & \rightarrow \frac{d_{2 \overline{2}}^{(l)}+d_{12 \overline{1} \overline{2}}^{(l)} E_{1} \bar{E}_{1}}{c_{2 \overline{2}}+c_{12 \overline{1} \overline{2}} E_{1} \bar{E}_{1}} \rho_{l}\left(\mathrm{i} a_{l}\right)^{n} e^{\omega_{l} t} \\
& \left.\rightarrow \frac{1}{2} \rho_{l} e^{\mathrm{i}\left(\zeta_{l}+2 \mathrm{i} \phi_{l}^{(2)}\right.}\right)\left(1+e^{2 \mathrm{i} \phi_{l}^{(1)}}+\left(e^{2 \mathrm{i} \phi_{l}^{(1)}}-1\right) \tanh \left(\xi_{1 R}+\xi_{0}^{1+}\right)\right),
\end{aligned}
$$

where

$$
\begin{aligned}
& A_{j}^{1+}=\frac{c_{12 \overline{2}}^{(j)}}{2 \sqrt{c_{1 \overline{1}}} \sqrt{c_{12 \overline{1} \overline{2}}}}, \quad e^{2 \xi_{0}^{1+}}=\frac{c_{12 \overline{1} \overline{2}}}{c_{2 \overline{2}}}, \\
& e^{2 \mathrm{i} \phi_{l}^{(2)}}=-\frac{\left(p_{2}-a_{l}\right)\left(\bar{p}_{2}-a_{l}\right)}{\left(1-a_{l} p_{2}\right)\left(1-a_{l} \bar{p}_{2}\right)}, \quad \zeta_{l}=n \varphi_{l}+n \pi / 2+2 \phi_{l}^{(2)}+\omega_{l} t,
\end{aligned}
$$

Soliton $2\left(\eta_{2 R} \approx 0, \eta_{1 R} \rightarrow-\infty\right)$ :

$$
\begin{aligned}
& q_{n}^{(j)} \rightarrow \frac{\alpha_{2}^{(j)} \mathrm{i}^{n} E_{2}}{1+c_{2 \overline{2}} E_{2} \bar{E}_{2}} \rightarrow A_{j}^{2+} e^{\mathrm{i} \xi_{2 I}} \operatorname{sech}\left(\xi_{2 R}+\xi_{0}^{2+}\right), \\
& q_{n}^{(l+m)} \rightarrow \frac{1+d_{2 \overline{2}}^{(l)} E_{2} \bar{E}_{2}}{1+c_{2 \overline{2}} E_{2} \bar{E}_{2}} \rho_{l}\left(\mathrm{i} a_{l}\right)^{n} e^{\omega_{l} t} \rightarrow \frac{1}{2} \rho_{l} e^{\mathrm{i} \zeta_{l}}\left(1+e^{2 \mathrm{i} \phi_{l}^{(2)}}+\left(e^{2 \mathrm{i} \phi_{l}^{(2)}}-1\right) \tanh \left(\xi_{2 R}+\xi_{0}^{2+}\right)\right),
\end{aligned}
$$

where

$$
A_{j}^{2+}=\frac{\alpha_{2}^{(j)}}{2 \sqrt{c_{2} \overline{2}}}, \quad e^{2 \xi_{0}^{2+}}=c_{2 \overline{2}} .
$$

\section{Acknowledgements}

We greatly appreciate all referees' useful comments which help us improve the present paper significantly. The work of B.F. is partially supported by NSF Grant (No. 1715991) and the COS Research Enhancement Seed Grants Program at UTRGV. The work of Y.O. is partly supported by JSPS Grant-in-Aid for Scientific Research (B-24340029, S-24224001, C-15K04909) and for Challenging Exploratory Research (26610029).

\section{References}

[1] Ablowitz M.J., Biondini G., Prinari B., Inverse scattering transform for the integrable discrete nonlinear Schrödinger equation with nonvanishing boundary conditions, Inverse Problems 23 (2007), 1711-1758.

[2] Ablowitz M.J., Clarkson P.A., Solitons, nonlinear evolution equations and inverse scattering, London Mathematical Society Lecture Note Series, Vol. 149, Cambridge University Press, Cambridge, 1991.

[3] Ablowitz M.J., Ladik J.F., Nonlinear differential-difference equations, J. Math. Phys. 16 (1975), 598-603. 
[4] Ablowitz M.J., Ladik J.F., Nonlinear differential-difference equations and Fourier analysis, J. Math. Phys. 17 (1976), 1011-1018.

[5] Ablowitz M.J., Ohta Y., Trubatch A.D., On discretizations of the vector nonlinear Schrödinger equation, Phys. Lett. A 253 (1999), 287-304, solv-int/9810014.

[6] Ablowitz M.J., Prinari B., Trubatch A.D., Discrete and continuous nonlinear Schrödinger systems, London Mathematical Society Lecture Note Series, Vol. 302, Cambridge University Press, Cambridge, 2004.

[7] Adler V.E., Postnikov V.V., On vector analogs of the modified Volterra lattice, J. Phys. A: Math. Gen. 41 (2008), 455203, 16 pages, arXiv:0808.0101.

[8] Agrawal G.P., Nonlinear fiber optics, 5th ed., Elsevier Inc., London, 2013.

[9] Barashenkov I.V., Getmanov B.S., Multisoliton solutions in the scheme for unified description of integrable relativistic massive fields. Non-degenerate $\mathfrak{s l}(2, \mathbb{C})$ case, Comm. Math. Phys. 112 (1987), 423-446.

[10] Barashenkov I.V., Getmanov B.S., Kovtun V.E., Integrable model with nontrivial interaction between suband superluminal solitons, Phys. Lett. A 128 (1988), 182-186.

[11] Barashenkov I.V., Getmanov B.S., Kovtun V.E., The unified approach to integrable relativistic equations: soliton solutions over nonvanishing backgrounds. I, J. Math. Phys. 34 (1993), 3039-3053.

[12] Barashenkov I.V., Getmanov B.S., The unified approach to integrable relativistic equations: soliton solutions over nonvanishing backgrounds. II, J. Math. Phys. 34 (1993), 3054-3072.

[13] Benney D.J., Newell A.C., The propagation of nonlinear wave envelopes, Stud. Appl. Math. 46 (1967), $133-139$.

[14] Dalfovo F., Giorgini S., Pitaevskii L.P., Stringari S., Theory of Bose-Einstein condensation in trapped gases, Rev. Modern Phys. 71 (1999), 463-512, cond-mat/9806038.

[15] Doliwa A., Santini P.M., Integrable dynamics of a discrete curve and the Ablowitz-Ladik hierarchy, J. Math. Phys. 36 (1995), 1259-1273, solv-int/9407005.

[16] Dubrovin B.A., Malanyuk T.M., Krichever I.M., Makhan'kov V.G., Exact solutions of the time-dependent Schrödinger equation with self-consistent potentials, Sov. J. Part. Nucl. 19 (1988), 252-269.

[17] Feng B.-F., General $N$-soliton solution to a vector nonlinear Schrödinger equation, J. Phys. A: Math. Theor. 47 (2014), 355203, 22 pages.

[18] Hasegawa A., Kodama Y., Solitons in optical communications, Clarendon, Oxford, 1995.

[19] Hasegawa A., Tappert F., Transmission of stationary nonlinear optical pulses in dispersive dielectric fibers. I. Anomolous dispersion, Appl. Phys. Lett. 23 (1973), 142-144.

[20] Hasegawa A., Tappert F., Transmission of stationary nonlinear optical pulses in dispersive dielectric fibers. II. Normal dispersion, Appl. Phys. Lett. 23 (1973), 171-172.

[21] Hirota R., The direct method in soliton theory, Cambridge Tracts in Mathematics, Vol. 155, Cambridge University Press, Cambridge, 2004.

[22] Kanna T., Lakshmanan M., Tchofo Dinda P., Akhmediev N., Soliton collisions with shape change by intensity redistribution in mixed coupled nonlinear Schrödinger equations, Phys. Rev. E 73 (2006), 026604, 15 pages, nlin.SI/0511034.

[23] Kivshar Y.S., Agrawal G.P., Optical solitons: from fibers to photonic crystals, Academic Press, San Diego, 2003.

[24] Krökel D., Halas N.J., Giuliani G., Grischkowsky D., Dark-pulse propagation in optical fibers, Phys. Rev. Lett. 60 (1988), 29-32.

[25] Makhan'kov V.G., Pashaev O.K., Nonlinear Schrödinger equation with noncompact isogroup, Theoret. and Math. Phys. 121 (1982), 979-987.

[26] Manakov S.V., On the theory of two-dimensional stationary self-focusing of electromagnetic waves, Sov. Phys. JETP 38 (1974), 248-253.

[27] Maruno K.-I., Ohta Y., Casorati determinant form of dark soliton solutions of the discrete nonlinear Schrödinger equation, J. Phys. Soc. Japan 75 (2006), 054002, 10 pages, nlin.SI/0506052.

[28] Narita K., Soliton solution for discrete Hirota equation, J. Phys. Soc. Japan 59 (1990), 3528-3530.

[29] Ohta Y., Pfaffian solution for coupled discrete nonlinear Schrödinger equation, Chaos Solitons Fractals 11 (2000), 91-95. 
[30] Ohta Y., Special solutions of discrete integrable systems, in Discrete Integrable Systems, Lecture Notes in Phys., Vol. 644, Editors B. Grammaticos, Y. Kosmann-Schwarzbach, T. Tamizhmani, Springer, Berlin, 2004, $57-83$.

[31] Ohta Y., Discretization of coupled nonlinear Schrödinger equations, Stud. Appl. Math. 122 (2009), $427-447$.

[32] Ohta Y., Hirota R., Tsujimoto S., Imai T., Casorati and discrete Gram type determinant representations of solutions to the discrete KP hierarchy, J. Phys. Soc. Japan 62 (1993), 1872-1886.

[33] Ohta Y., Wang D.-S., Yang J., General N-dark-dark solitons in the coupled nonlinear Schrödinger equations, Stud. Appl. Math. 127 (2011), 345-371, arXiv:1011.2522.

[34] Park Q.-H., Shin H.J., Systematic construction of multicomponent optical solitons, Phys. Rev. E 61 (2000), 3093-3106.

[35] Prinari B., Ablowitz M.J., Biondini G., Inverse scattering transform for the vector nonlinear Schrödinger equation with nonvanishing boundary conditions, J. Math. Phys. 47 (2006), 063508, 33 pages.

[36] Prinari B., Vitale F., Inverse scattering transform for the focusing Ablowitz-Ladik system with nonzero boundary conditions, Stud. Appl. Math. 137 (2016), 28-52.

[37] Radhakrishnan R., Lakshmanan M., Bright and dark soliton solutions to coupled nonlinear Schrödinger equations, J. Phys. A: Math. Gen. 28 (1995), 2683-2692.

[38] Sheppard A.P., Kivshar Y.S., Polarized dark solitons in isotropic Kerr media, Phys. Rev. E 55 (1997), $4773-4782$.

[39] Tsuchida T., Integrable discretization of coupled nonlinear Schrödinger equations, Rep. Math. Phys. 46 (2000), 269-278, nlin.SI/0002048.

[40] Tsuchida T., Ujino H., Wadati M., Integrable semi-discretization of the coupled nonlinear Schrödinger equations, J. Phys. A: Math. Gen. 32 (1999), 2239-2262, solv-int/9903013.

[41] Tsujimoto S., Integrable discretization of the integrable systems, in Applied Integrable Systems, Editor Y. Nakamura, Shokabo, Tokyo, 2000, 1-52.

[42] Tsuzuki T., Nonlinear waves in the Pitaevskii-Gross equation, J. Low Temp. Phys. 4 (1971), 441-457.

[43] van der Mee C., Inverse scattering transform for the discrete focusing nonlinear Schrödinger equation with nonvanishing boundary conditions, J. Nonlinear Math. Phys. 22 (2015), 233-264.

[44] Vekslerchik V.E., Konotop V.V., Discrete nonlinear Schrödinger equation under nonvanishing boundary conditions, Inverse Problems 8 (1992), 889-909.

[45] Vijayajayanthi M., Kanna T., Lakshmanan M., Bright-dark solitons and their collisions in mixed $N$-coupled nonlinear Schrödinger equations, Phys. Rev. A $\mathbf{7 7}$ (2008), 013820, 18 pages, arXiv:0711.4424.

[46] Weiner A.M., Heritage J.P., Hawkins R.J., Thurston R.N., Kirschner E.M., Leaird D.E., Tomlinson W.J., Experimental observation of the fundamental dark soliton in optical fibers, Phys. Rev. Lett. 61 (1988), 2445-2448.

[47] Yajima N., Oikawa M., A class of exactly solvable nonlinear evolution equations, Progr. Theoret. Phys. 54 (1975), 1576-1577.

[48] Zakharov V.E., Collapse of Langumuir waves, Sov. Phys. JETP 35 (1972), 908-914.

[49] Zakharov V.E., Shabat A.B., Exact theory of two-dimensional self-focusing and one-dimensional selfmodulation of waves in nonlinear media, Sov. Phys. JETP 34 (1972), 62-69.

[50] Zakharov V.E., Shabat A.B., Interaction betweem solitons in a stable medium, Sov. Phys. JETP 37 (1973), 823-828.

[51] Zhang Y.J., Cheng Y., Solutions for the vector k-constrained KP hierarchy, J. Math. Phys. 35 (1994), 5869-5884. 